\title{
Independencia funcional en la recuperación de pacientes sometidos a cirugía cardiaca.
}

\author{
Functional Independence in the recovery of patients with cardiac surgery. \\ Independência funcional na reabilitação de pacientes submetidos à cirurgia cardíaca \\ Jhon Henry Osorio-Castaño*
Juan Pablo Garcia-Carvajal
Marcela Gutierrez-Londoño
}

\section{Resumen}

Objetivo: Evaluar el nivel de independencia funcional de los pacientes sometidos a cirugía cardiaca durante su proceso de recuperación. Materiales y Método: Investigación con enfoque cuantitativo y estudio de tipo analítico longitudinal prospectivo. La población de estudio estuvo conformada por personas mayores de 18 años, sometidas a cirugía cardiaca programada, en una institución de salud de la ciudad de Medellín, Colombia. Se aplicó un cuestionario para las variables sociodemográficas y el procedimiento quirúrgico, además se utilizó el instrumento de independencia funcional de Granger y Hamilton traducido al español por Paolinelli. Se realizó un análisis univariado, bivariado y se usó la regresión de la mediana como prueba multivariada no paramétrica. Resultados: El total de participantes fue de 33. El 50\% tenía 64 años. El 66.7\% fueron hombres. Se encontró que el 57.6\% eran hipertensos y el $12.1 \%$ diabéticos. El $54.5 \%$ se sometió a bypass coronario. Se evidencia una diferencia estadísticamente significativa entre cada una de las mediciones de la independencia funcional $(\mathrm{p}<0.05)$ y una relación directa entre la edad y la disminución de la autonomía ( $p$ 0.034). Conclusiones: La independencia funcional se afecta tras la realización de la cirugía cardiaca, pero se recupera con el paso del tiempo y está de alguna manera relacionada con la edad.

Palabras clave: Actividades Cotidianas, Cirugía Torácica, Evaluación de la Discapacidad.

\section{Abstract}

Objective: Evaluate the level of functional independence in patients during their recovery from cardiac surgery. Materials and Method: Quantitative focus research and analytical longitudinal prospective study type. The population of study consisted of people older than 18 , subjected to scheduled cardiac surgery, in a health institution in the city of Medellin, Colombia. A questionnaire was applied for the sociodemographic variables and the surgical procedure, the Granger and Hamilton instrument of

\section{Autor de correspondencia}

* $\triangle$ Enfermero. Especialista en Gerencia de IPS. Magister en Epidemiología. Docente. Universidad Pontificia Bolivariana. Email: jhon.osorio@upb.edu.co. Orcid: https://orcid.org/0000-0001-6272-722X. Medellín, Colombia.

** Enfermero. Especialista en Enfermería Cardiovascular. Clínica Cardiovid. Email: juapalo10@hotmail.com. Orcid: https:// orcid.org/0000-0001-7414-2341. Medellín, Colombia.

*** Enfermera. Especialista en Enfermería Cardiovascular. Clínica Cardiovid. Email: gutilond@yahoo.es. Orcid: https://orcid.org/00000002-5081-6679. Medellín, Colombia. 
ISSN-PRINT

1794-9831

E-ISSN 2322-7028

Vol. 16 No. 2

May - Ago 2019

Cúcuta, Colombia

functional independence was also used (translated to Spanish by Paolinelli). Univariate and bivariate analysis were made, and the median regression was used as non-parametric multivariate proof. Results: The total participants of the study were $33.50 \%$ of them were 64 years old. $66.7 \%$ were men. It was found that $57.6 \%$ were hypertense and $12.2 \%$ had diabetes. $54.5 \%$ of the participants were subjected to coronary bypass surgery. A statistically significant difference is evidenced between each of the measures of functional independence $(\mathrm{p}<0.005)$, and a direct relationship between age and loss of autonomy ( $\mathrm{p}$ 0.034). Conclusions: Functional independence is affected through cardiac surgery, but it is recovered with time, and it is somehow related with age.

Key words: Daily activities, Cardiothoracic surgery, Evaluation of disability.

\section{Resumo}

Objetivo: Avaliar o nível de independência funcional dos pacientes submetidos à cirurgia cardíaca durante o seu processo de reabilitação. Materiais e métodos: Estudo quantitativo de tipo analítico, longitudinal e prospectivo. A população estudada foram pessoas maiores de 18 anos, submetidas a cirurgia cardíaca programada, numa instituição de saúde na cidade de Medellin, Colômbia. Aplicouse o questionário para as variáveis sociais, demográficas e do procedimento cirúrgico. Utilizou-se o instrumento de independência funcional de Granger e Hamilton traduzido ao espanhol por Paolinelli. Realizou-se uma análise univariada e bivariada e usou-se a regressão da mediana como teste multivariado não paramétrico. Resultados: Foram estudados 33 pacientes 50\% tinham 64 anos de idade y $66,7 \%$ eram do sexo masculino. Encontrou-se que 57,6\% tinham hipertensão e 12,1\% Diabetes Mellitus. 54,5\% se submeteu a revascularização coronariana. Observou-se diferença estatística significativa entre cada medição de independência funcional $(\mathrm{p}<0,05)$ e uma relação direta entre a idade e a diminuição da autonomia $(\mathrm{p}=0,034)$. Conclusões: A independência funcional encontra-se afetada pós cirurgia cardíaca, porém pode-se recuperar com o decorrer do tempo, também está relacionada com a idade.

Palavras-chave: Atividades cotidianas, cirurgia torácica, reabilitação, pessoas com deficiência

\section{Introducción}

Cada año mueren más personas por enfermedad cardiovascular que por cualquier otra causa, de acuerdo con los datos de la Organización Mundial de la Salud (1). En Colombia, según informes del Ministerio de Protección Social entre los años 2005 y 2013, la principal causa de muerte en la población en general fueron las enfermedades del sistema circulatorio; aunque se siguió una tendencia descendente con el paso del tiempo, pasando de 166,42 a 146,16 muertes por cada 100.000 habitantes; es decir, que causaron el $29,92 \%$ (529.190) de las defunciones y el 16,13\% (7.016.697) de todos los Años de Vida Potencialmente Perdidos (AVPP) (2).

La cirugía cardiaca se ha enfocado en impactar la morbilidad y la mortalidad de los eventos cardiovasculares, proporcionando a los pacientes nuevas alternativas de tratamiento; se han utilizado nuevas técnicas quirúrgicas que han disminuido los índices de mortalidad operatoria contribuyendo al aumento de la esperanza de vida de pacientes que han visto afectadas diversas dimensiones de su vida por el curso de la enfermedad (3). Dentro del tratamiento quirúrgico se pueden encontrar: cirugías valvulares, revascularización coronaria, cirugías combinadas (valvulares y coronarias), cirugías de cardiopatías congénitas y cirugías vasculares.

Sin embargo, los pacientes sometidos a cirugía cardiaca experimentan una serie de alteraciones que afectan sus funciones fisiológicas y de la vida diaria. Las primeras, son provocadas por la circulación extracorpórea, los fenómenos de isquemia-reperfusión en el corazón, la hipotermia, trastornos en el sistema de coagulación y los efectos adversos de las transfusiones y el sangrado (4); y las segundas, debido a inmovilización por tiempo prolongado, que se asocia con el síndrome de desacondicionamiento físico o debilidad aguda del paciente crítico; siendo una de sus consecuencias, la disminución en la capacidad funcional que, sumada a la enfermedad de base, los compromete en forma multisistémica (5).

El proceso de recuperación de las funciones fisiológicas y de la vida diaria, varían de acuerdo con el estado previo y las características propias de cada paciente. En algunos casos, como en pacientes ancianos o pa- 
cientes con comorbilidades previas, se requerirá mayor tiempo para la restauración de las funciones mencionadas, lo que implica mayores cuidados o cuidados más prolongados, siendo un factor importante, el tiempo trascurrido entre la cirugía cardiaca y la restauración de la independencia funcional de los pacientes (6).

Por otra parte, el aumento de tiempo en la estancia de cuidado intensivo se asocia con mayor compromiso en la movilidad articular, la fuerza muscular y la capacidad funcional (7), dando origen a repercusiones significativas en las prácticas de autocuidado de cada individuo que de acuerdo con Orem (8) se definen, como la práctica de las actividades que las personas inician y llevan a cabo en determinados periodos, con el interés de mantener un funcionamiento vivo y sano, y así continuar con el desarrollo personal y el bienestar, mediante la satisfacción de requisitos que faciliten las regulaciones funcional y del desarrollo. Se debe tener en cuenta que estos requisitos son característicos de cada persona y se asocian con su periodo de crecimiento y desarrollo, su estado de salud, características específicas de la salud o estados de desarrollo, nivel de desgaste de energía o factores medioambientales.

De igual forma, se plantea en la teoría del déficit de autocuidado que la edad, el desarrollo y el estado de salud pueden afectar la capacidad que tenga la persona para realizar las actividades de la vida diaria (8). $\mathrm{Si}$ bien es cierto, que el referente teórico que orientó este estudio no fue el Marco de Autocuidado de Dorothea Orem, se puede indicar que las propuestas derivadas de las teorías del autocuidado, del déficit de autocuidado y de los sistemas de enfermería, pueden contribuir en la comprensión del proceso de recuperación de la independencia funcional y aunque este concepto no hace parte de la propuesta de Orem, se puede pensar que la autonomía sea un objetivo de los sistemas de enfermería para lograr un mejor autocuidado en los pacientes sometidos a cirugía cardíaca.

La independencia funcional se entiende como la capacidad de un individuo para llevar a cabo actividades de la vida diaria para mantener el cuerpo y subsistir independientemente (9). La pérdida de independencia funcional que se da como consecuencia de una cirugía cardiaca y una inmovilidad prolongada requiere una evaluación previa, debido a su pronóstico, convirtiéndose en un fuerte predictor de los resultados de salud para el estado funcional y la supervivencia $(10,11)$.
Se han propuesto diferentes escalas para medir la independencia funcional, entre ellas están: el Índice de Katz (12), validado en idiomas como el portugués (13) y el turco (14) y el Índice de Barthel (15) validado en idiomas como el italiano (16) y el iraní (17). Cada escala busca valorar las actividades básicas de la vida diaria y suelen ser aplicadas en diferentes contextos del proceso salud - enfermedad. La característica común de estas escalas es que evalúan sólo las personas con respecto a su desempeño en las actividades motoras, sin evaluar, por ejemplo, las habilidades de comunicación o los factores cognitivos y sociales (18). En contraste, la Medida de Independencia Funcional (FIM), desarrollada por Linacre et al. (19) fue diseñada para medir la independencia/dependencia, enfocándose en dos dominios principales: el cognitivo y el motor.

Por ser la enfermedad cardiovascular la principal causa de morbilidad y mortalidad en el mundo y la cirugía cardíaca una de las principales alternativas terapéuticas; es importante que la enfermería tenga conocimiento acerca de la evolución de la independencia funcional de los pacientes que son sometidos a intervenciones quirúrgicas y sus factores relacionados, de tal forma que posteriormente, puedan ser formuladas intervenciones que promuevan esta independencia, en el marco de programas o protocolos institucionales y domiciliarios de acompañamiento multidisciplinar y que a su vez favorezcan la autonomía profesional del enfermero.

\section{Objetivos}

\section{Objetivo General}

Evaluar el nivel de independencia funcional de los pacientes sometidos a cirugía cardíaca durante su proceso de recuperación.

\section{Objetivos específicos}

- Describir la independencia funcional antes y después de la intervención quirúrgica.

- Comparar los niveles de independencia funcional en diferentes momentos del proceso de recuperación.

- Identificar los factores relacionados con la recuperación de la independencia funcional. 
ISSN-PRINT

$1794-9831$

E-ISSN 2322-7028

Vol. 16 No. 2

May - Ago 2019

Cúcuta, Colombia

\section{Materiales y Método}

La presente investigación tuvo un enfoque cuantitativo de tipo analítico longitudinal prospectivo. Se realizó en una institución de salud de alta complejidad que presta servicios para personas con eventos cardiovasculares en la ciudad de Medellín, en el periodo comprendido entre junio y agosto de 2017. El tiempo de recolección de la información estuvo determinado por los costos del estudio y la disponibilidad de la institución, factores que determinaron las características de la muestra. Los escenarios de aplicación de los instrumentos incluyeron consulta externa, cuidados intensivos y hospitalización.

La población objeto de estudio estuvo conformada por personas mayores de 18 años sometidas a cirugía cardiaca programada. No hubo cálculo de muestra, porque la frecuencia de pacientes con cirugía cardíaca programada en esta institución es relativamente baja y por la disponibilidad de la institución para la realización del estudio; de igual forma, se seleccionaron todos los pacientes que cumplieron con los criterios de inclusión y fueron atendidos en el periodo estipulado, por tanto, tampoco hubo muestreo.

Se eligieron los pacientes que iban a ser sometidos a cirugía de bypass coronario, cambio o plastia valvular, cirugía mixta: bypass coronario + cambio valvular y cirugías congénitas tipo cierre de comunicación interauricular (CIA), cierre de comunicación interventricular (CIV) y cambio o plastia de aorta bivalva. Se excluyeron los pacientes que presentaban negativa o incapacidad para dar el consentimiento informado, trasplante cardiaco o pulmonar, sometidos a cirugía urgente, con secuelas mayores de ACV (paresias, paraplejía, hemiplejía, cuadriplejía), pacientes con dependencia para las actividades de la vida diaria, con enfermedad pulmonar obstructiva crónica Gold D y pacientes internacionales.

Se diseñó un cuestionario que contenía las siguientes variables sociodemográficas y clínicas: edad, sexo, peso, talla, índice de masa corporal, nivel de escolaridad, estado civil, estrato socioeconómico, ocupación, número de personas con quien vive, antecedentes personales (HTA, DM, enfermedad renal crónica, EPOC, cáncer, falla cardiaca), cirugía cardiaca previa; y para las variables relacionadas con el procedimiento quirúrgico: tipo de cirugía cardiaca, uso de circulación extracorpórea, tiempo de pinza, tiempo de perfusión, realización de esternotomía, re-intervención en menos de 24 horas, días de estancia en UCI, días de estancia en hospitalización.

Los pacientes que fueron sometidos a cirugía cardiaca y cumplieron con los criterios de inclusión se abordaron durante la hospitalización, 3 días previos a la cirugía o durante la consulta programada por enfermería; se les invitó a participar del estudio y si aceptaban se diligenciaba el consentimiento informado. Adicionalmente, se le informó al acompañante acerca de la participación del paciente en la investigación, debido a que, en las mediciones posteriores, era probable que se necesitara de su ayuda. La primera medición se hizo antes de la cirugía, con el objetivo de recolectar la información socio-demográfica y clínica; también se aplicó el instrumento para medir la independencia funcional. Posteriormente se hicieron tres mediciones más: la segunda en el momento de egreso de la unidad de cuidados intensivos, instante en el que también, se recolectaron las variables relacionadas con el procedimiento quirúrgico; la tercera medición, en el momento del egreso hospitalario y la cuarta durante la consulta de revisión con el cirujano cardiovascular.

Para medir la variable desenlace se utilizó como instrumento de evaluación la Medida de Independencia Funcional de Linacre et al. (19) traducida al español por Paolinelli et al. (20), estudio que se realizó con 100 pacientes ingresados a un programa de rehabilitación y con niveles de concordancia inter-observador de 0.67 a 0.82 en personal entrenado, comparado con concordancias de 0.33 a 0.76 en personal no entrenado; siendo esta la única medida calculada debido a que el instrumento es dependiente del observador. El uso del instrumento no requirió solicitud de permisos ya que se encuentra disponible de manera libre.

La Medida de Independencia Funcional es una escala que evalúa dos dominios: motor y cognitivo; fue construida a partir de siete niveles de funcionalidad, dos que no requieren de la ayuda humana y cinco en los que se necesita un grado progresivo de ayuda. Se compone de 6 áreas de funcionamiento, para las cuales se han definido 18 ítems de evaluación constituidos por actividades de la vida diaria: autocuidado (alimentación, arreglo personal, baño, vestido cuerpo superior, vestido cuerpo inferior, aseo perineal), control de esfinteres (control de la vejiga, control del intestino), movilidad (traslado de la cama/silla, traslado en baño, traslado a la ducha), deambulación (caminar o desplazarse en silla de ruedas, 
subir y bajar escaleras), comunicación (comprensión , expresión), y conciencia del mundo exterior (interacción social, resolución de problemas, memoria).

La máxima puntuación de cada ítem es de siete y la mínima de uno, divididos en tres grandes grupos así:

- Nivel de funcionalidad independiente. Se asigna un puntaje de 7 para completamente independiente y 6 independiente con cierta limitación.

- Nivel de funcionalidad dependencia modificada. Se subdivide en supervisión a la cual se le asigna un valor de 5; para mínima asistencia 4, moderada asistencia 3 .

- Nivel de funcionalidad dependencia completa. Se asigna un puntaje de 2 para asistencia máxima y 1 punto para total asistencia. Al totalizar la escala el máximo puntaje obtenido será de 126 y el mínimo de 18.

Las fuentes de información fueron el paciente y la historia clínica. El instrumento para la recolección de la información y la escala de independencia funcional fue administrado por los investigadores previo entrenamiento clínico en su uso. Se creó una base de datos en Excel para almacenar todos los datos obtenidos a través de su validación y control.

Para los diferentes análisis se definió como variable desenlace la medida de independencia funcional. Esta variable se manejó de forma cuantitativa para algunos análisis y de forma cualitativa ordinal para otros.

Se realizó un análisis univariado aplicando una distribución porcentual a las variables cualitativas y a las cuantitativas, promedio y desviación estándar o mediana y rango inter-cuartílico, dependiendo de si los datos presentaban una distribución normal de acuerdo con la prueba de Shapiro-Wilk. Para describir la capacidad funcional se utilizó una distribución porcentual para cada categoría aplicando la siguiente fórmula: $\left(\mathrm{N}^{\circ}\right.$ de pacientes con cada nivel de independencia/Total de pacientes evaluados) $* 100$.

De modo similar, se realizó una comparación entre las medidas de independencia funcional con la prueba no paramétrica de Wilcoxon. Se comparó la independencia funcional en la segunda medición y en la cuarta medición de acuerdo al tipo de cirugía y estrato socio-económico a través de la prueba no paramétrica de Kruskall-Wallis.
Posteriormente, se realizó una comparación de las medidas de independencia funcional en el momento 2 y 4 con las variables dicotómicas de sexo, presencia de co-morbilidad, circulación extra-corpórea, esternotomía, cirugía previa y re-intervención con el estadístico U de Mann-Whitney. Así mismo, se procedió a realizar una regresión de la mediana como prueba multivariante no paramétrica con aquellas variables que se reportaron como significativas. Para todos los análisis se tomó como valor significativo una $\mathrm{p}<0.05$ y se ejecutó teniendo en cuenta los aplicativos Epidat versión 3.5, SPSS versión 20 y Stata versión 11 suministrados por la universidad.

El estudio fue aprobado por el Comité de Ética de Investigación de la Universidad Pontificia Bolivariana según consta en el acta $\mathrm{N}^{\circ} 4$ del 27 de marzo del 2017. De igual forma, fue aprobado por el Comité de Ética de la institución hospitalaria donde se llevó a cabo el proyecto. Todos los participantes firmaron el consentimiento informado respectivo y se garantizó el resguardo de la confidencialidad y privacidad de la información recolectada mediante el anonimato de las encuestas e instrumentos aplicados.

\section{Resultados}

El total de participantes del estudio fueron 33. El 50\% tenía 64 años y más, con un rango de 14 años. El 66.7\% fueron hombres, el $18 \%$ con secundaria incompleta y $15.2 \%$ con pregrado; $36.4 \%$ pertenecientes al estrato socioeconómico 3 , casados en un $60.6 \%$ y pensionados en un $24.2 \%$. El $36.4 \%$ de los participantes convivían con dos personas. Respecto a los antecedentes personales patológicos se encontró que el 57.6\% eran hipertensos, $12.1 \%$ diabéticos, $3 \%$ tenían insuficiencia renal crónica, $12.1 \%$ insuficiencia cardíaca congestiva, 3\% cáncer y enfermedad arterial oclusiva crónica.

En relación con el tipo de cirugía realizada, el 54.5\% fue sometido a bypass coronario, seguido de cambio valvular en un $15.2 \%$. En el $90.9 \%$ de los participantes se utilizó circulación extracorpórea y el $66.7 \%$ se le sometió a esternotomía. Solo el $6.1 \%$ fueron reintervenidos en las primeras 24 horas; $57.5 \%$ estuvieron en la unidad de cuidados intensivos un día, 21.2\% dos días; el tiempo de hospitalización fue de 2 días para el $36.4 \%$, seguido de 3 días para el $21.2 \%$.

El promedio del peso fue de $70.9 \mathrm{~kg}$ (DE 12.1), el de la talla de $1.64 \mathrm{~cm}$ (DE 0.89) y el índice de masa corporal
E-ISSN 2322-7028

Vol. 16 No. 2

May - Ago 2019

Cúcuta, Colombia 
ISSN-PRINT

1794-9831

E-ISSN 2322-7028

Vol. 16 No. 2

May - Ago 2019

Cúcuta, Colombia de 25.9 (DE 3.0). E1 57.6\% de los participantes tenían sobrepeso. La media del tiempo de pinza fue de 72 minutos (DE 38.3) y la del tiempo de perfusión 96.4 minutos (DE 53.2). Como puede apreciarse en la tabla
1, un alto porcentaje de participantes presentaron independencia completa en los ítems evaluados antes de la cirugía cardíaca.

Tabla 1. Distribución porcentual como resultados de la independencia funcional en los pacientes, previo a la cirugía cardíaca.

\begin{tabular}{|c|c|c|c|c|c|c|c|}
\hline Ítem & 7 & 6 & 5 & 4 & 3 & 2 & 1 \\
\hline Alimentación & $94 \%$ & $6 \%$ & & & & & \\
\hline Aseo personal & $97 \%$ & $3 \%$ & & & & & \\
\hline Baño & $97 \%$ & & & & & & $3 \%$ \\
\hline Vestirse cuerpo superior & $97 \%$ & & & & & & $3 \%$ \\
\hline Vestirse cuerpo inferior & $97 \%$ & & & & & & $3 \%$ \\
\hline Aseo perineal & $97 \%$ & & & & $3 \%$ & & \\
\hline Control vesical & $94 \%$ & & & & & & $6 \%$ \\
\hline Control intestinal & $100 \%$ & & & & & & \\
\hline Traslado cama-silla & $94 \%$ & $3 \%$ & & & & & $3 \%$ \\
\hline Traslado baño & $94 \%$ & $3 \%$ & & & & & $3 \%$ \\
\hline Traslado ducha & $97 \%$ & & & & & & $3 \%$ \\
\hline Marcha & $91 \%$ & $3 \%$ & $3 \%$ & & & & $3 \%$ \\
\hline Uso escaleras & $91 \%$ & $6 \%$ & & & & & $3 \%$ \\
\hline Comprensión & $97 \%$ & & $3 \%$ & & & & \\
\hline Expresión & $97 \%$ & $3 \%$ & & & & & \\
\hline Interacción social & $97 \%$ & & $3 \%$ & & & & \\
\hline Solución problemas & $97 \%$ & & & & $3 \%$ & & \\
\hline Memoria & $82 \%$ & $18 \%$ & & & & & \\
\hline
\end{tabular}

Fuente: Elaboración propia.

El 50\% de los participantes obtuvieron un puntaje de 55 y menos en la escala de independencia funcional con un rango de 45 después de la cirugía cardíaca. Al revisar cada ítem, el $6.1 \%$ tuvo total asistencia para la alimentación, el $45.5 \%$ para el aseo personal, el $75.8 \%$ para el baño, el $90.9 \%$ para vestirse, el $69.7 \%$ para el aseo perineal, el $93.9 \%$ para control vesical e intestinal; $57.6 \%$ presentó dependencia completa para traslado a camilla y $97 \%$ total asistencia para traslado a baño y ducha.

El $57.6 \%$ tuvo total asistencia para la marcha y $100 \%$ para subir escaleras; solo 3\% requirió mínima asistencia, supervisión e independencia con limitación respectivamente para la comprensión y la expresión; el 3\% tuvo supervisión y $6.2 \%$ el mismo resultado para interacción social; en cuanto al ítem de solución de problemas, el 3\% necesitó de mínima asistencia y dependencia completa para la memoria en el mismo porcentaje.

Al comparar la mediana del nivel de independencia previo y posterior a la cirugía se evidencia una di- ferencia estadísticamente significativa (p 0.000); esta misma situación se observa en la comparación entre el nivel de independencia posterior a la cirugía y el egreso de la UCI (p 0.000) y entre el egreso de la UCI y el alta hospitalaria (p 0.000).

De igual forma, se demostró que existe una diferencia entre la independencia funcional al egreso de UCI y la cita de revisión con el especialista (p 0.000); y entre la independencia previo a la cirugía y la cita de revisión con el especialista ( $\mathrm{p}$ 0.017).

Al analizar el resultado de la independencia funcional relacionado con otras variables, se comprobó que no hubo diferencias en el nivel de independencia después de la cirugía y una semana después de la hospitalización por estrato socioeconómico (p 0.35), ni tipo de cirugía (p 0.55). Al comparar por sexo, comorbilidades, uso de circulación extra-corpórea, esternotomía, cirugía previa y re-intervención entre ambos momentos de la medición no se evidenciaron diferencias significativas. 
Tabla 2. Regresión de la mediana para la edad y la independencia funcional en la cita de revisión con el cirujano cardiovascular.

\begin{tabular}{ccccccc}
\hline Ind_Fun_4 & Coef. & Std. Err. & $\mathbf{t}$ & $\mathbf{P}>|\mathbf{t}|$ & \multicolumn{2}{c}{$[\mathbf{9 5 \%}$ Conf. Interval] } \\
\hline Edad & $-0,0588236$ & 0,0252173 & $-2,33$ & 0,026 & $-0,1102546$ & $-0,0073926$ \\
_cons & 128,4118 & 1,563172 & 82,15 & 0 & 125,2237 & 131,5999 \\
\hline
\end{tabular}

Fuente: Elaboración Propia

Para indagar sobre las posibles correlaciones de los valores obtenidos en la última medición de la independencia funcional que correspondía a la cita de revisión con el cirujano cardiovascular, con otras variables cuantitativas, se procedió a realizar una regresión de la mediana como prueba no paramétrica debido a la ausencia de distribución normal. Inicialmente, se ingresó al modelo la edad dando como resultado un $\beta$ de -0.058 (IC95\% -0.110 a -0.007 ) y un valor p 0.026 (Ver tabla 2). Lo que indica que, a mayor edad, menor resultado en la última medida de la independencia funcional; cuando se ajusta por otras variables como peso, talla, tiempo de pinza y tiempo de perfusión, la edad continúa siendo significativa con un $\beta$ de -0.08 (IC95\% -0.16 a -0.007 ) y un valor p 0.034 (Ver tabla 3).

Tabla 3. Regresión de la mediana para la edad, el peso, la talla, tiempo de pinza, tiempo de perfusión y la independencia funcional en la cita de revisión con el cirujano cardiovascular.

\begin{tabular}{lllcrrr}
\hline \multicolumn{1}{c}{ Ind_Fun_4 } & Coef. & Std. Err. & \multicolumn{1}{c}{ t } & \multicolumn{1}{c}{$\mathbf{P}>|\mathbf{t}|$} & \multicolumn{2}{c}{ [95\% Conf. Interval] } \\
\hline Peso & 0,0436907 & 0,0515879 & 0,85 & 0,404 & $-0,0621589$ & 0,1495403 \\
Talla & 0,0469602 & 7,078904 & 0,01 & 0,995 & $-14,47775$ & 14,57167 \\
Tiempo pinza & 0,0192635 & 0,0302965 & 0,64 & 0,53 & $-0,0428997$ & 0,0814267 \\
Tiempo de pen & $-0,0163035$ & 0,0203889 & $-0,80$ & 0,431 & $-0,0581381$ & 0,0255312 \\
Edad & $-0,086658$ & 0,0387699 & $-2,24$ & 0,034 & $-0,1662072$ & $-0,0071088$ \\
_Cons & 127,0238 & 9,586018 & 13,25 & 0,000 & 107,3549 & 146,6926 \\
\hline
\end{tabular}

Fuente: Elaboración Propia

\section{Discusión}

La enfermedad cardiovascular es la primera causa de morbimortalidad en Colombia y en el mundo; su tratamiento se basa en terapias farmacológicas y en fases avanzadas mediante tratamiento quirúrgico o intervencionista. Los pacientes que son sometidos a cirugía cardiaca atraviesan una serie de alteraciones que afectan sus funciones fisiológicas y las motoras y/o cognitivas, lo que conlleva a una disminución en la capacidad para desarrollar las actividades de la vida diaria, generando así, un déficit de autocuidado $(21,22)$. Adicionalmente, la escala utilizada en este estudio es útil para medir el estado funcional de las personas cuando son dadas de alta de un servicio de cuidado intensivo (23).

En este estudio se evaluó el nivel de independencia funcional de los pacientes sometidos a cirugía cardiaca durante su proceso de recuperación, encontrando diferencias estadísticamente significativas entre los diferentes momentos del proceso de recuperación (UCI, Hospitalización, Egreso, Revisión), seguidos desde el preoperatorio hasta la evaluación final; resultados equiparables con el estudio realizado por Niemeyer et al. (4) donde se muestra una disminución importante en el estado funcional después de la cirugía cardiaca con una lenta recuperación en el tiempo, además se demostró una asociación positiva entre la edad y los valores de la escala de medida de independencia funcional.

Situación que pone de manifiesto la necesidad de implementar sistemas de enfermería totalmente compensatorios hasta que los pacientes recuperen su independencia funcional y al lograrlo, recurrir a un sistema de apoyo educativo, tal como lo propone Orem en su teoría de los sistemas de enfermería (8).

Es necesario aclarar que este estudio no evaluó in- 
ISSN-PRINT

1794-9831

E-ISSN 2322-7028

Vol. 16 No. 2

May - Ago 2019

Cúcuta, Colombia tervenciones de ningún tipo en la recuperación de la independencia funcional de los pacientes; por lo que las alusiones al Marco de Autocuidado de Dorothea Orem, solo responden a una integración teórica de lo que podría ser la contribución de la enfermería a la asistencia de estos pacientes sometidos a cirugía cardíaca; de ahí, que se evidencie la necesidad de cuidado directo y totalmente compensado para suplir sus necesidades básicas.

Del mismo modo, se evidenció que durante la medición posterior a la cirugía, los pacientes presentaban mayor dependencia para la ejecución de sus actividades de la vida diaria explicado por las limitaciones para la movilización tales como: accesorios de uso terapéutico (sondas, drenes, catéteres, vía aérea artificial), inestabilidad hemodinámica y respiratoria (24). También se debe precisar, que el cuidado intensivo postoperatorio de la cirugía cardíaca, tiene como objetivo la recuperación de la homeostasis que se ve afectada por los cambios fisiopatológicos generados por el uso de la circulación extracorpórea, los fenómenos de isquemia-reperfusión en el corazón, la hipotermia, trastornos en el sistema de coagulación y los efectos adversos de las transfusiones y el sangrado $(23,25)$; por tanto, el reposo en cama puede conllevar al desacondicionamiento físico (26).

La edad avanzada se convirtió en la variable que más influyó en la recuperación de los pacientes sometidos a cirugía cardíaca, medida con la independencia funcional. Estudios como el de Tahiri et al. (6) y el de Laar et al. (27) confirmaron cómo la edad avanzada afecta no solo la recuperación del estado funcional, sino que también incide en las complicaciones postoperatorias. Adicionalmente, otros estudios (28, 29) han demostrado que la edad de los sujetos sometidos a cirugía cardiaca, bien sea por enfermedad coronaria, recambio valvular aórtico o mitral, se ha ido elevando progresivamente en el curso de las últimas décadas, siendo los mayores de 60 años el grupo en quienes las enfermedades crónicas cardiovasculares tienen mayor incidencia, debido a un crecimiento demográfico de ésta población. Es precisamente, en este tipo de pacientes, en quienes la independencia se encuentra disminuida posterior a la cirugía, debido a una reserva funcional más baja, secundaria tal vez, a la presencia de más comorbilidades, lo que los hace más propensos a sufrir complicaciones (30).

En este estudio se identificó que no hubo diferencias en el nivel de independencia funcional de los pacientes después de la cirugía relacionándola con la variable de uso de circulación extra-corpórea diferente a lo encontrado por Curzel et al. (6) quienes demuestran que a mayor tiempo de circulación extracorpórea menor puntaje en la escala de medida de independencia funcional; esto puede estar relacionado con la poca variabilidad en los tiempos de cirugía,así como al desconocimiento por parte de los profesionales sobre los efectos negativos de la circulación extracórporea, que pueden tener incidencia en el acto quirúrgico y por ende en el menor tiempo de exposición a este procedimiento (31).

La presencia de enfermedades crónicas distintas de las enfermedades cardiovasculares no afectó los puntajes de la independencia funcional. Este resultado contrasta con el estudio realizado por Karakurt et al. (32) con pacientes ancianos, donde se encontró que los pacientes se volvieron más dependientes para las actividades de la vida diaria a medida que aumentaba el número de enfermedades crónicas.

Con respecto al ítem de subir escaleras se demostró que todos los pacientes requerían de una asistencia total para realizar este tipo de ejercicio después de la cirugía y del alta hospitalaria, relacionándose directamente con el protocolo de rehabilitación cardiaca de la institución donde se realizó la investigación, el cual recomienda no realizar esta actividad física hasta no completar por lo menos 5 días posquirúrgicos, además de su relación con la corta estancia hospitalaria promediada en tres días. Este resultado es igual a otro estudio (33) donde se recomienda el inicio de la actividad física, basada en ejercicios musculares básicos y sencillos y bajo la supervisión de profesionales entrenados y especializados. Esto se puede explicar porque después de la cirugía cardíaca, la demanda cardiopulmonar es mayor y por tanto regula o limita la movilidad, siendo este factor un componente fundamental para el desarrollo de la actividad física en estos pacientes (7).

En cuanto a la valoración de los ítems compresión, expresión, interacción social, solución de problemas y memoria, se encontraron cambios significativos comparando la valoración previa a la cirugía y posterior a ésta, lo cual se equipara con el estudio de Raffa et al. (34) donde se describe que en el posoperatorio inmediato se observan alteraciones intelectuales en más del $80 \%$ de los pacientes, siendo incierto su me- 
canismo de patogenia; éste fenómeno se ha relacionado con la hipoperfusión sistémica y embolización peri operatoria, variables que no se tuvieron en cuenta en este estudio; sin embargo, se debe mencionar que ninguno de los participantes en el experimento de sintomatología asociada a delirium, tuviesen problemas relacionados con la dimensión cognitiva, cuando fueron sometidos a cirugía y hospitalización en unidades de cuidados intensivos $(35,36)$.

Dentro de las limitaciones del estudio se puede mencionar que se contó con una muestra pequeña, lo cual puede relacionarse con un período corto de recolección de la información; por otro lado, los criterios de inclusión no permitieron seleccionar algunos de los pacientes que fueron sometidos a cirugía cardiaca durante este tiempo. De igual forma, la frecuencia de cirugía cardíaca en la institución no es alta.

Se recomienda ampliar el tiempo del estudio y redefinir los criterios de inclusión de los pacientes sometidos a cirugía cardiaca, debido a que la enfermedad cardiovascular es la primera causa de morbimortalidad a nivel mundial y local. Es necesario evaluar la independencia funcional de los pacientes sometidos a cirugía cardiaca y diseñar programas de acompañamiento o de restablecimiento de la misma por medio de intervenciones que involucren la compensación completa del cuidado hasta llegar a actividades de apoyo educativo. Intervenciones que deben involucrar a la familia como principal red de apoyo y al cuidador principal; pero, además, se debe fomentar la educación sobre prácticas de autocuidado que contri- buyan a mantener en el mejor estado de salud posible al paciente receptor de la cirugía cardiovascular. Además, se debe tener en cuenta la identificación de factores que se relacionen con el proceso de recuperación de la independencia funcional, puesto que favorece la formulación de estrategias que impacten a otras funciones o actividades como el retorno a la vida laboral, la interacción familiar, o en general las actividades básicas de la vida diaria.

\section{Conclusiones}

- La independencia funcional se afecta tras la realización de cirugía cardiaca, pero se recupera con el paso del tiempo y probablemente, esta recuperación esté de alguna manera explicada por la edad. La movilización precoz del paciente es fundamental para su pronta recuperación, de tal forma que vaya adquiriendo de manera progresiva su autonomía y así recuperar sus capacidades motoras, dándole de esta forma independencia.

- A la luz de estos hallazgos, se debe alentar a las enfermeras a diseñar programas educativos y de capacitación que incluyan servicios de atención para que los pacientes puedan adquirir independencia en las actividades de la vida diaria durante el período postoperatorio.

\section{Conflicto de Intereses}

Los autores declaran no tener ningún conflicto de intereses

\section{Referencias bibliográficas}

1. World Health Organization. Global Status Report on noncommunicable diseases 2014 [Internet]. 2014. [Consultado 2017 jun 15]. Disponible en: http://apps.who.int/iris/bitstre am/10665/148114/1/9789241564854 eng.pdf?ua $=1$

2. Ministerio de Salud. Análisis de situación de salud. Colombia. 2015 [Internet]. 2016 [Consultado 2017 jun 19]. Disponible en: https://www.minsalud.gov.co/sites/rid/Lists/BibliotecaDigital/RIDE/VS/ED/ PSP/asis-2015.pdf

3. Romero PS, Noguiera de Souza E, Rodrigues J, Moraes MA. Changes in quality of life associated with surgical risk in elderly patients undergoing cardiac surgery. Int J Nurs Pract. 2015;21(5):592-7.

4. Niemeyer Guimarães M, Cendoroglo M, Almada Filho CM. Course of functional status in elderly patients after coronary artery bypass surgery: 6-month follow up. Geriatr Gerontol Int [Internet]. 2016 [Consultado 2017 jun 19]; 16(6):737-46. Disponible en: https://doi.org/10.1111/ggi.12547

5. Barrie K, Cornick A, Debreuil S, Lee E, Hiebert M, Manji R, et al. Patients with a prolonged intensive care unit lenght of stay have decreased health-related quality of life after cardiac surgery. Semin Thoracic Surg [Internet]. 2018 [Consultado 2019 feb 28]; 31(1):21-31. Disponible en: https://doi. 
ISSN-PRINT

1794-9831

E-ISSN 2322-7028

Vol. 16 No. 2

May - Ago 2019

Cúcuta, Colombia org/10.1053/j.semtcvs.2018.07.005

6. Tahiri M, Sikder T, Maimon G, Teasdale D, Hamadani F, Sourial N, et al. The impact of postoperative complications on the recovery of elderly surgical patients. Surg Endosc Other Interv Tech. [Internet]. 2016 [Consultado 2017 jul 19]; 30(5):1762-70. Disponible en: https://doi: 10.1007/s00464-015-4440-2

7. Miranda Rocha AR, Martinez BP, Maldaner da Silva VZ, Forgiarini Junior LA. Early mobilization: Why, what for and how? Med Intensiva [Internet]. 2017 [Consultado 2017 jul 19]; 41(7):429-36. Disponible en: http://dx.doi.org/10.1016/j.medin.2016.10.003

8. Prado Solar LA, González Reguera M, Paz Gómez N, Romero Borges K. La teoría Défícit de autocuidado: Dorothea Orem punto de partida para calidad en la atención. Rev Médica Electrónica [Internet]. 2014 [Consultado 2017 jul 19]; 36(6):835-45. Disponible en: http://scielo.sld.cu/scielo. php?script $=$ sci_arttext\&pid $=$ S1684-18242014000600004

9. de Jesus F, Paim D, Brito J, Barros I, Barbosa T, Prata B, et al. Mobility decline in patients hospitalized in an intensive care unit. Rev Bras Ter Intensiva. 2016; 28(2):114-19.

10. Hyum D, Kim C, Placide S, Lipsitz L, Marcantonio E. Preoperative frailty assessment and outcomes at 6 months or later in older adults undergoing cardiac surgical procedures. Ann Intern Med [Internet]. 2016 [Consultado 2019 feb 28]; 165:650-60. Disponible en: https://www.ncbi.nlm.nih.gov/ pubmed/27548070

11. Chiapinotto S, Dallazen F, Teixeira Bodnar E, Winkelmann ER. Nível de dor e grau de independência funcional de pacientes submetidos à cirurgia cardíaca. Rev Baiana Enfermagemi [Internet]. 2017 [Consultado 2017 jul 19]; 31(4):1-8. Disponible en: https://portalseer.ufba.br/index.php/enfermagem/ article/view/21388

12. Katz S, Downs T, Cash H, Grotz R. Progress in Development of the Index of ADL. Gerontologist. [Internet]. 1970 [Consultado 2017 may 10]; 10(1):20-30. Disponible en: https://www.ncbi.nlm.nih. gov/pubmed/5420677

13. Saraiva Lino VT, Mendes Pereira SR, Bastos Camacho LA, Ribeiro Filho ST, Buksman S. Adaptação transcultural da Escala de Independência em Atividades da Vida Diária (Escala de Katz). Cad Saude Pública [Internet]. 2008 [Consultado 2017 may 10]; 24(1):103-12. Disponible en: http://dx.doi. org/10.1590/S0102-311X2008000100010

14. Arik G, Varan HD, Yavuz BB, Karabulut E, Kara O, Kilic MK, et al. Validation of Katz index of independence in activities of daily living in Turkish older adults. Arch Gerontol Geriatr [Internet]. 2015 [Consultado 2017 may 10]; 61(3):344-50. Disponible en: http://dx.doi.org/10.1016/j.archger.2015.08.019

15. Mahoney FI, Wood OH, Barthel DW. Rehabilitation of chronically ill patients: the influence of complications on the final goal. South Med J. [Internet]. 1958 [Consultado 2017 may 10]; 51(5):605-9. Disponible en: https://www.ncbi.nlm.nih.gov/pubmed/13556165

16. Castiglia SF, Galeoto G, Lauta A, Palumbo A, Tirinelli F, Viselli F, et al. The culturally adapted Italian version of the Barthel Index (IcaBI): assessment of structural validity, inter-rater reliability and responsiveness to clinically relevant improvements in patients admitted to inpatient rehabilitation centers. Funct Neurol. [Internet].2017 [Consultado 2017 may 10]; 32(4):221-8. Disponible en: https:// www.ncbi.nlm.nih.gov/pubmed/29306359

17. Oveisgharan S, Shirani S, Ghorbani A, Soltanzade A, Baghaei A, Hosseini S, et al. Barthel index in a Middle-East country: Translation, validity and reliability. Cerebrovasc Dis. [Internet] 2006 [Consultado 2017 may 10];22(5-6):350-4. Disponible en: https://www.karger.com/Article/Abstract/94850

18. Prodinger B, O'Connor RJ, Stucki G, Tennant A. Establishing score equivalence of the functional independence measure motor scale and the barthel index, utilizing the international classification of functioning, disability and health and rasch measurement theory. J Rehabil Med. [Internet] 2017 [Consultado 2017 may 10]; 49(5):416-22. Disponible en: https://www.ncbi.nlm.nih.gov/pubmed/28471470 
19. Linacre J, Heinemann A, Wright B, Granger C, Hamilton B. The structure and stability of the Functional Independence Measure. Arch Phys Med Rehabil. [Internet] 1994 [Consultado 2017 may 10]; 75(2):127-32. Disponible en: https://www.archives-pmr.org/article/0003-9993(94)90384-0/fulltext

20. Paolinelli C, González P, Doniez ME, Donoso T, Salinas V. Instrumento de evaluación funcional de la discapacidad en rehabilitación.: Estudio de confiabilidad y experiencia clínica con el uso del Functional Independence Measure. Rev Med Chil. 2001; 129(1):1-10

21. Miwa S, Visintainer P, Engelman R, Miller A, Lagu T, Woodbury E, et al. Effects of an Ambulation Orderly Program Among Cardiac Surgery Patients. Am J Med [Internet]. 2017 [Consultado 2017 jul 15]; 130(11):1306-12. Disponible en: https://doi.org/10.1016/j.amjmed.2017.04.044

22. Leahy JM, Hoagland B, Strange RG, Antevil JL. Effects of Cardiac Surgery on Duty Status in the Active Duty Military Population. Mil Med [Internet]. 2015 [Consultado 2017 jul 20];180(7):792-7. Disponible en: https://www.ncbi.nlm.nih.gov/pubmed/26126251

23. Serpytis P, Navickas P, Navickas A, Serpytis R, Navickas G, Glaveckaite S. Age-and gender-related peculiarities of patients with delirium in the cardiac intensive care unit. Kardiol Pol [Internet]. 2017 [Consultado 2019 feb 28];75(10):1041-50. Disponible en: https://www.ncbi.nlm.nih.gov/ pubmed/28715077

24. Ferrão C, Quintaneiro C, Camila C, Aragão I, Cardoso T. Evaluation of long-term outcomes of very old patients admitted to intensive care: Survival, functional status, quality of life, and quality-adjusted life-years. J Crit Care [Internet]. 2015 [Consultado 2017 ago 23];30(5):1150.e7-1150.e11. Disponible en: http://dx.doi.org/10.1016/j.jcrc.2015.05.005

25. Hall M, Robertson J, Merkel M, Aziz M, Hutchens M. A structured transfer of care process reduces perioperative complications in cardiac surgery patients. Anesth Analg [Internet]. 2017 [Consultado 2019 feb 28]; 125(2):477-82. Disponible en: https://www.ncbi.nlm.nih.gov/pubmed/28504990

26. Pintado MC, Villa P, Luján J, Trascasa M, Molina R, González-García N, et al. Mortalidad y estado funcional al año de pacientes ancianos con ingreso prolongado en una unidad de cuidados intensivos. Med Intensiva. 2016;40(5):289-97.

27. van Laar C, Timman ST, Noyez L. Decreased physical activity is a predictor for a complicated recovery post cardiac surgery. Health Qual Life Outcomes [Internet]. 2017 [Consultado 2017 sep 12];15(5):1-7. Disponible en: http://dx.doi.org/10.1186/s12955-016-0576-6

28. Etayo EH, Gonzalez FI, Florián MC, Chacon A. Cirugia cardiaca en ancianos. Epidemiología, calidad de vida y funcionalidad postoperatoria. Acta Med Colomb. 2014;39(2):118-23.

29. Rich M, Skolnick A, Alexander K, Forman D, Kitzman D, Maurer M, et al. Knowledge gaps in cardiovascular care of the older adult population: a scientific statement from the American Heart Association, American College of Cardiology, and American Geriatrics Society. J Am Coll Cardiol [Internet]. 2016 [Consultado 2019 feb 28];67(20):2419-40. Disponible en: https://doi.org/10.1016/j.jacc.2016.03.004

30. Seco M, Edelman J, Forrest P, Martin N, Wilson M, Fraser J, et al. Geriatric cardiac surgery: chronology vs. biology. Heart, Lung and Circulation [Internet]. 2014 [Consultado 2019 feb 28]; 23:794-01. Disponible en: https:// doi.org/10.1016/j.hlc.2014.04.008

31. Freundt M, Ried M, Philipp A, Diez C, Kolat P, Hirt S, et al. Minimized extracorporeal circulation is improving outcome of coronary artery bypass surgery in the elderly. Perfusion [Internet]. 2016 [Consultado 2017 nov 24]; 31(2):143-8. Disponible en: https://doi.org/10.1177/0267659115588634

32. Karakurt P, Kasimoğlu N, Bahçeli A, Atalikoğlu Başkan S, Ağdemir B. The effect of activities of daily living on the self-care agency of patients in a cardiovascular surgery clinic. J Vasc Nurs. [Internet] 2017 [Consultado 2017 nov 24]; 35(2):78-85. Disponible en: https://doi.org/10.1016/j.jvn.2016.10.004

33. Seo YG, Jang MJ, Park WH, Hong KP, Sung J. Inpatient cardiac rehabilitation programs exercise therapy for patients undergoing cardiac surgery : National korean questionnaire survey. J Excercise Rehabil. [Internet] 2017 [Consultado 2017 dic 09]; 13(1):76-83. Disponible en: https://doi.org/10.12965/jer.1732806.403 
ISSN-PRINT

$1794-9831$

E-ISSN 2322-7028

Vol. 16 No. 2

May - Ago 2019

Cúcuta, Colombia
34. Raffa G, Agnello F, Occhipinti G, Miraglia R, Lo Re V, Marrone G, et al. Neurological complications after cardiac surgery: a retrospective case-control study of risk factors and outcome. [Internet] 2019 [Consultado 2019 feb 28]; 14(23):1-10. Disponible en: https://doi.org/10.1186/s13019-019-0844-8

35. Delaney A, Hammond N, Litton E. Preventing Delirium in the Intensive Care Unit. JAMA. 2018;319(7):659-60.

36. Evans AS, Weiner MM, Arora RC, Chung I, Deshpande R, Varghese R, et al. Current approach to diagnosis and treatment of delirium after cardiac surgery. Ann Card Anaesth [Internet]. 2016 [Consultado 2017 dic 09]; 19(2):328-37. Disponible en: http:/www.annals.in/text.asp?2016/19/2/328/179634 